\title{
A simultaneous iterative method for split equality problems of two finite families of strictly pseudononspreading mappings without prior knowledge of operator norms
}

Haitao Che ${ }^{*}$ and Meixia Li

"Correspondence:

haitaoche@163.com

School of Mathematics and

Information Science, Weifang

University, Weifang, Shandong

261061, China

\begin{abstract}
In this article, we first introduce the concept of $T$-mapping of a finite family of strictly pseudononspreading mapping $\left\{T_{i}\right\}_{i=1}^{N}$, and we show that the fixed point set of the $T$-mapping is the set of common fixed points of $\left\{T_{i}\right\}_{i=1}^{N}$ and $T$ is a quasi-nonexpansive mapping. Based on the concept of a $T$-mapping, we propose a simultaneous iterative algorithm to solve the split equality problem with a way of selecting the stepsizes which does not need any prior information about the operator norms. The sequences generated by the algorithm weakly converge to a solution of the split equality problem of two finite families of strictly pseudononspreading mappings. Furthermore, we apply our iterative algorithms to some convex and nonlinear problems.
\end{abstract}

MSC: 47H09; 47H05; 47H06; 47J25

Keywords: fixed point; feasibility problem; simultaneous iterative method; weak convergence; strictly pseudononspreading mapping

\section{Introduction}

Due to their extraordinary utility and broad applicability in many areas of applied mathematics (most notably, fully discretized models of problems in image reconstruction from projections, in image processing, and in intensity-modulated radiation therapy), algorithms for solving convex feasibility problems continue to receive great attention; see for instance [1-11]. Recently, Moudafi [12] introduced a new convex feasibility problem (CFP). Let $H_{1}, H_{2}, H_{3}$ be real Hilbert spaces, let $C \subset H_{1}, Q \subset H_{2}$ be two nonempty closed convex sets, let $A: H_{1} \rightarrow H_{3}, B: H_{2} \rightarrow H_{3}$ be two bounded linear operators. The convex feasibility problem in [12] is to find

$$
x \in C, y \in Q \text { such that } A x=B y,
$$

which allows asymmetric and partial relations between the variables $x$ and $y$. The interest is to cover many situations, for instance in decomposition methods for PDEs, applications in game theory and in intensity-modulated radiation therapy (IMRT). In decision sciences, this allows one to consider agents who interplay only via some components of their decision variables, for further details, the interested reader is referred to [13]. In IMRT, this

(อ2015 Che and Li; licensee Springer. This is an Open Access article distributed under the terms of the Creative Commons Attribution License (http://creativecommons.org/licenses/by/2.0), which permits unrestricted use, distribution, and reproduction in any medium, provided the original work is properly cited. 
amounts to envisage a weak coupling between the vector of doses absorbed in all voxels and that of the radiation intensity, for further details, the interested reader is referred to $[13,14]$.

For solving the CFP (1.1), Moudafi [12] studied the fixed point formulation of the solutions of the CFP (1.1). Assume that the CFP (1.1) is consistent (i.e., (1.1) has a solution), if $(x, y)$ solves (1.1), then it solves the following fixed point equation system:

$$
\left\{\begin{array}{l}
x=P_{C}\left(x-\gamma A^{*}(A x-B y)\right), \\
y=P_{Q}\left(y+\beta B^{*}(A x-B y)\right),
\end{array}\right.
$$

where $\gamma, \beta>0$ are any positive constants, and then Moudafi introduced the following alternating $C Q$ algorithm:

$$
\left\{\begin{array}{l}
x_{k+1}=P_{C}\left(x_{k}-\gamma_{k} A^{*}\left(A x_{k}-B y_{k}\right)\right), \\
y_{k+1}=P_{Q}\left(y_{k}+\beta_{k} B^{*}\left(A x_{k+1}-B y_{k}\right)\right),
\end{array}\right.
$$

where $\gamma_{k}, \beta_{k} \in\left(\varepsilon, \min \left(\frac{1}{\lambda_{A}}, \frac{1}{\lambda_{B}}\right)-\varepsilon\right), \lambda_{A}$ and $\lambda_{B}$ are the spectral radii of $A^{*} A$ and $B^{*} B$, respectively. The weak convergence of the sequence $\left(x_{k}, y_{k}\right)$ to a solution of (1.1) under some conditions was proved.

In [15], Moudafi and Al-Shemas considered the following problem:

$$
x \in F(U), y \in F(T) \text { such that } A x=B y,
$$

and proposed the following simultaneous algorithm:

$$
\left\{\begin{array}{l}
x_{k+1}=U\left(x_{k}-\gamma_{k} A^{*}\left(A x_{k}-B y_{k}\right)\right), \\
y_{k+1}=T\left(y_{k}+\gamma_{k} B^{*}\left(A x_{k}-B y_{k}\right)\right),
\end{array}\right.
$$

for firmly quasi-nonexpansive operators $U$ and $T$, where $\gamma_{k} \in\left(\varepsilon, \frac{2}{\lambda_{A}+\lambda_{B}},-\varepsilon\right), \lambda_{A}$ and $\lambda_{B}$ are the spectral radiuses of $A^{*} A$ and $B^{*} B$, respectively.

Observe that in the algorithms (1.3) and (1.5) mentioned above, the determination of the stepsize $\left\{\gamma_{k}\right\}$ depends on the operator (matrix) norms $\|A\|$ and $\|B\|$ (or the largest eigenvalues of $A^{*} A$ and $B^{*} B$ ). To implement the alternating algorithm (1.3) and the simultaneous algorithm (1.5), one has first to compute (or, at least, estimate) operator norms of $A$ and $B$, which is in general not easy in practice.

To overcome this difficulty, Lopez et al. [16] and Zhao et al. [17] presented useful method for choosing the stepsizes which do not need prior knowledge of the operator norms for solving the split feasibility problems and multiple-set split feasibility problems, respectively.

Motivated by above results, we introduce a new choice of the stepsize sequence $\left\{\gamma_{k}\right\}$ for the simultaneous iterative algorithm to solve (1.4) governed by quasi-nonexpansive mapping as follows:

$$
\gamma_{k} \in\left(0, \min \left\{\frac{\left\|A x_{k}-B y_{k}\right\|^{2}}{\left\|A^{*}\left(A x_{k}-B y_{k}\right)\right\|^{2}}, \frac{\left\|A x_{k}-B y_{k}\right\|^{2}}{\left\|B^{*}\left(A x_{k}-B y_{k}\right)\right\|^{2}}\right\}\right) .
$$

The advantage of our choice (1.6) of the stepsizes lies in the fact that no prior information about the operator norms of $A$ and $B$ is required, and still convergence is guaranteed. 
In this article, we propose the following simultaneous iterative algorithm where the stepsizes do not depend on the operator norms $\|A\|$ and $\|B\|$ and prove the weak convergence of the algorithm to solve (1.4). Let $U: H_{1} \rightarrow H_{1}$ and $T: H_{2} \rightarrow H_{2}$ be two quasinonexpansive mappings which are defined by $(2.5)$. We denote by $\Gamma$ be the set of solutions of (1.4), i.e.,

$$
\Gamma=\{x \in F(U), y \in F(T) \text { such that } A x=B y\} .
$$

Algorithm 1.1 Let $x_{0} \in H_{1}, y_{0} \in H_{2}$ be arbitrary and $\left\{a_{k}\right\}$ be real number sequences in $[a, b] \subset(0,1)$. Assume that the $k$ th iterate $x_{k} \in H_{1}, y_{k} \in H_{2}$ has been constructed and $A x_{k}-$ $B y_{k} \neq 0$, then we calculate $(k+1)$ th iterate $\left(x_{k+1}, y_{k+1}\right)$ via the formula

$$
\left\{\begin{array}{l}
u_{k}=x_{k}-\gamma_{k} A^{*}\left(A x_{k}-B y_{k}\right), \\
x_{k+1}=a_{k} x_{k}+\left(1-a_{k}\right) U\left(u_{k}\right), \\
v_{k}=y_{k}+\gamma_{k} B^{*}\left(A x_{k}-B y_{k}\right), \\
y_{k+1}=a_{k} y_{k}+\left(1-a_{k}\right) T\left(v_{k}\right),
\end{array}\right.
$$

where the stepsize $\gamma_{k}$ is chosen by (1.6). If $A x_{k}-B y_{k}=0$, then $\left(x_{k}, y_{k}\right)=\left(x_{k+1}, y_{k+1}\right)$ is a solution of the problem (1.4) and the iterative process stops. Otherwise, we set $k:=k+1$ and go on to (1.7) to evaluate the next iterate $\left(x_{k+2}, y_{k+2}\right)$.

Remark 1.1 Notice that in (1.6) the choice of the stepsize $\gamma_{k}$ is independent of the norms $\|A\|$ and $\|B\|$.

\section{Preliminaries}

Throughout this paper, we denote by $H$ be a real Hilbert space with inner product $\langle\cdot, \cdot\rangle$ and induced norm $\|\cdot\|$, and denote by $C$ be a nonempty closed convex subset of $H$. Let $T: H \rightarrow H$ be a mapping. A point $x \in H$ is said to be a fixed point of $T$ provided $x=T x$. we use $F(T)$ to denote the fixed point set. We write $x_{n} \rightarrow x$ to indicate that the sequence $\left\{x_{n}\right\}$ converges weakly to $x, x_{n} \rightarrow x$ implies that $\left\{x_{n}\right\}$ converges strongly to $x$. We use $\omega_{w}\left(x_{k}\right)=$ $\left\{x: \exists x_{k_{j}} \rightarrow x\right\}$ to stand for the weak $\omega$-limit set of $\left\{x_{k}\right\}$. For any $x \in H$, there exists a unique nearest point in $C$, denoted by $P_{C} x$, such that

$$
\left\|x-P_{C} x\right\| \leq\|x-y\|, \quad \forall y \in C .
$$

Before proceeding, we need to introduce a few concepts.

A mapping $T: C \rightarrow C$ belongs to the set $\Phi_{q}$ of quasi-nonexpansive, if

$$
\|T x-q\| \leq\|x-q\|, \quad \forall(x, q) \in C \times F(T) .
$$

A mapping $T: C \rightarrow C$ belongs to the set $\Phi_{n}$ of nonexpansive, if

$$
\|T x-T y\| \leq\|x-y\|, \quad \forall(x, y) \in C \times C .
$$

A mapping $T: C \rightarrow C$ belongs to the set $\Phi_{f}$ of firmly nonexpansive, if

$$
\|T x-T y\|^{2} \leq\|x-y\|^{2}-\|(x-y)-(T x-T y)\|^{2}, \quad \forall(x, y) \in C \times C .
$$


A mapping $T: C \rightarrow C$ belongs to the set $\Phi_{f q}$ of firmly quasi-nonexpansive, if

$$
\|T x-q\| \leq\|x-q\|-\|x-T x\|^{2}, \quad \forall(x, q) \in C \times F(T) .
$$

A mapping $T: C \rightarrow C$ is called nonspreading, if

$$
\|T x-T y\|^{2} \leq\|x-y\|^{2}+2\langle x-T x, y-T y\rangle, \quad \forall x, y \in K .
$$

A mapping $T: C \rightarrow C$ is called $k$-strictly pseudononspreading if there exists $k \in[0,1)$ such that

$$
\|T x-T y\|^{2} \leq\|x-y\|^{2}+k\|x-T x-(y-T y)\|^{2}+2\langle x-T x, y-T y\rangle, \quad \forall x, y \in C .
$$

Remark 2.1 It is easy to see that $\Phi_{f} \subset \Phi_{n} \subset \Phi_{q}$ and $\Phi_{f} \subset \Phi_{f q} \subset \Phi_{q}$. Furthermore, $\Phi_{f}$ is well known to contain resolvents and projection operators, and $\Phi_{f q}$ includes subgradient projection operators [18]. $T$ is a nonspreading mapping if and only if $T$ is a 0 -strictly pseudononspreading mapping.

The so-called demiclosedness principle plays an important role in our argument.

A mapping $T: H \rightarrow H$ is called demiclosed at the origin if for any sequence $\left\{x_{n}\right\}$ which weakly converges to $x$, and if the sequence $\left\{x_{n}\right\}$ strongly converges to 0 , then $T x=0$.

To establish our results, we need the following technical lemmas.

Lemma 2.1 ([19]) If $x, y, z \in H$, then:

(a) $\|x+y\|^{2} \leq\|x\|^{2}+2\langle y, x+y\rangle$.

(b) For any $\lambda \in[0,1]$,

$$
\|\lambda x+(1-\lambda) y\|^{2}=\lambda\|x\|^{2}+(1-\lambda)\|y\|^{2}-\lambda(1-\lambda)\|x-y\|^{2} .
$$

(c) For $a, b, c \in[0,1]$ with $a+b+c=1$,

$$
\|a x+b y+c z\|^{2}=a\|x\|^{2}+b\|y\|^{2}+c\|z\|^{2}-a b\|x-y\|^{2}-a c\|x-z\|^{2}-b c\|y-z\|^{2} .
$$

The following definition will be useful for our results.

In 2009, Kangtunyakarn and Suantai [20] introduced $T$-mapping generated by $T_{1}, T_{2}$, $\ldots, T_{N}$ and $\lambda_{1}, \lambda_{2}, \ldots, \lambda_{N}$ as follows.

Definition 2.1 Let $C$ be a nonempty convex subset of real Banach space. Let $\left\{T_{i}\right\}_{i=1}^{N}$ be a finite family of mappings of $C$ into itself, and let $\lambda_{1}, \lambda_{2}, \ldots, \lambda_{N}$ be real numbers such that $0<\lambda_{i}<1$ for every $1,2, \ldots, N$. We define a mapping $T: C \rightarrow C$ as follows:

$$
\left\{\begin{array}{l}
U_{1}=\lambda_{1} T_{1}+\left(1-\lambda_{1}\right) I, \\
U_{2}=\lambda_{2} T_{2} U_{1}+\left(1-\lambda_{2}\right) U_{1}, \\
U_{3}=\lambda_{3} T_{3} U_{2}+\left(1-\lambda_{3}\right) U_{2}, \\
\ldots, \\
U_{N-1}=\lambda_{N-1} T_{N-1} U_{N-2}+\left(1-\lambda_{N-1}\right) U_{N-2}, \\
T=U_{N}=\lambda_{N} T_{N} U_{N-1}+\left(1-\lambda_{N}\right) U_{N-1} .
\end{array}\right.
$$

Such a mapping $T$ is called the $T$-mapping generated by $T_{1}, T_{2}, \ldots, T_{N}$ and $\lambda_{1}, \lambda_{2}, \ldots, \lambda_{N}$. 
Using the above definition, we have the following important lemma.

Lemma 2.2 Let C be a nonempty convex subset of real Banach space. Let $\left\{T_{i}\right\}_{i=1}^{N}$ be a finite family of $\rho_{i}$-strictly pseudononspreading mappings of $C$ into itself with $\bigcap_{i=1}^{N} F\left(T_{i}\right) \neq \emptyset$, and let $\lambda_{1}, \lambda_{2}, \ldots, \lambda_{N}$ be real numbers such that $0<\lambda_{i}+\rho_{i}<1$ for every $1,2, \ldots, N$. If $T$ is the $T$-mapping generated by $T_{1}, T_{2}, \ldots, T_{N}$ and $\lambda_{1}, \lambda_{2}, \ldots, \lambda_{N}$, then $F(T)=\bigcap_{i=1}^{N} F\left(T_{i}\right)$ and $T$ is a quasi-nonexpansive mapping.

Proof It is easy to deduce that $\bigcap_{i=1}^{N} F\left(T_{i}\right) \subset F(T)$. Next, we claim that $F(T) \subset \bigcap_{i=1}^{N} F\left(T_{i}\right)$. Let $x_{0} \in F(T)$ and $x^{*} \in \bigcap_{i=1}^{N} F\left(T_{i}\right)$. Assume that $U_{0}=I$, for $i=1,2, \ldots, N$, it follows from $\left\{T_{i}\right\}_{i=1}^{N}$ being a finite family of $\rho_{i}$-strictly pseudononspreading mappings of $C$ into itself that

$$
\begin{aligned}
&\left\langle U_{i-1} x_{0}-T_{i} U_{i-1} x_{0}, U_{i-1} x_{0}-x^{*}\right\rangle \\
&=\frac{1}{2}\left\|U_{i-1} x_{0}-T_{i} U_{i-1} x_{0}\right\|^{2}+\frac{1}{2}\left\|U_{i-1} x_{0}-x^{*}\right\|^{2}-\frac{1}{2}\left\|T_{i} U_{i-1} x_{0}-x^{*}\right\|^{2} \\
&=\frac{1-\rho_{i}}{2}\left\|U_{i-1} x_{0}-T_{i} U_{i-1} x_{0}\right\|^{2}-\frac{1}{2}\left\|T_{i} U_{i-1} x_{0}-x^{*}\right\|^{2} \\
& \quad+\frac{1}{2}\left(\left\|U_{i-1} x_{0}-x^{*}\right\|^{2}+\rho_{i}\left\|U_{i-1} x_{0}-T_{i} U_{i-1} x_{0}\right\|^{2}\right) \\
& \geq \frac{1-\rho_{i}}{2}\left\|U_{i-1} x_{0}-T_{i} U_{i-1} x_{0}\right\|^{2} .
\end{aligned}
$$

From the definition of $T$ and (2.6), we have

$$
\begin{aligned}
\left\|x_{0}-x^{*}\right\|^{2}= & \left\|T x_{0}-x^{*}\right\|^{2} \\
= & \left\|\lambda_{N} T_{N} U_{N-1} x_{0}+\left(1-\lambda_{N}\right) U_{N-1} x_{0}-x^{*}\right\|^{2} \\
= & \left\|\lambda_{N}\left(T_{N} U_{N-1} x_{0}-x^{*}\right)+\left(1-\lambda_{N}\right)\left(U_{N-1} x_{0}-x^{*}\right)\right\|^{2} \\
= & \lambda_{N}^{2}\left\|T_{N} U_{N-1} x_{0}-x^{*}\right\|^{2}+\left(1-\lambda_{N}\right)^{2}\left\|U_{N-1} x_{0}-x^{*}\right\|^{2} \\
& +2 \lambda_{N}\left(1-\lambda_{N}\right)\left\langle T_{N} U_{N-1} x_{0}-x^{*}, U_{N-1} x_{0}-x^{*}\right\rangle \\
= & \lambda_{N}^{2}\left\|T_{N} U_{N-1} x_{0}-x^{*}\right\|^{2}+\left(1-\lambda_{N}\right)^{2}\left\|U_{N-1} x_{0}-x^{*}\right\|^{2} \\
& +2 \lambda_{N}\left(1-\lambda_{N}\right)\left\langle T_{N} U_{N-1} x_{0}-U_{N-1} x_{0}, U_{N-1} x_{0}-x^{*}\right\rangle \\
& +2 \lambda_{N}\left(1-\lambda_{N}\right)\left\|U_{N-1} x_{0}-x^{*}\right\|^{2} \\
\leq & \left\|U_{N-1} x_{0}-x^{*}\right\|^{2}-\lambda_{N}\left[1-\left(\rho_{N}+\lambda_{N}\right)\right]\left\|U_{N-1} x_{0}-T_{N} U_{N-1} x_{0}\right\|^{2} \\
\leq & \left\|U_{N-1} x_{0}-x^{*}\right\|^{2} \\
& \cdots \\
\leq & \left\|U_{2} x_{0}-x^{*}\right\|^{2}-\lambda_{3}\left[1-\left(\rho_{3}+\lambda_{3}\right)\right]\left\|U_{2} x_{0}-T_{3} U_{2} x_{0}\right\|^{2} \\
\leq & \left\|U_{2} x_{0}-x^{*}\right\|^{2} \\
\leq & \left\|U_{1} x_{0}-x^{*}\right\|^{2}-\lambda_{2}\left[1-\left(\rho_{2}+\lambda_{2}\right)\right]\left\|U_{1} x_{0}-T_{2} U_{1} x_{0}\right\|^{2} \\
\leq & \left\|U_{1} x_{0}-x^{*}\right\|^{2} \\
\leq & \left\|x_{0}-x^{*}\right\|^{2}-\lambda_{1}\left[1-\left(\rho_{1}+\lambda_{1}\right)\right]\left\|x_{0}-T_{1} x_{0}\right\|^{2} \\
\leq & \left\|x_{0}-x^{*}\right\|^{2}, \\
&
\end{aligned}
$$


which means $\left\|x_{0}-T_{1} x_{0}\right\|=0$, that is, $x_{0} \in F\left(T_{1}\right)$. Furthermore,

$$
U_{1} x_{0}=\lambda_{1} T_{1} x_{0}+\left(1-\lambda_{1}\right) x_{0}=x_{0}
$$

it yields $x_{0} \in F\left(U_{1}\right)$. Applying the same argument, we can conclude that $x_{0} \in F\left(T_{i}\right)$ and $x_{0} \in F\left(U_{i}\right)$, for $i=1,2, \ldots, N-1$.

Next, we claim that $x_{0} \in F\left(T_{N}\right)$. Indeed,

$$
\begin{aligned}
0 & =T x_{0}-x_{0} \\
& =\lambda_{N} T_{N} U_{N-1} x_{0}+\left(1-\lambda_{N}\right) U_{N-1} x_{0}-x_{0} \\
& =\lambda_{N}\left(T_{N} x_{0}-x_{0}\right) .
\end{aligned}
$$

It follows that $x_{0} \in F\left(T_{N}\right)$. Therefore, $x_{0} \in \bigcap_{i=1}^{N} F\left(T_{i}\right)$, that is, $F(T) \subset \bigcap_{i=1}^{N} F\left(T_{i}\right)$. Hence, $F(T)=\bigcap_{i=1}^{N} F\left(T_{i}\right)$. From the definition of $T$ and (2.7), we find that $T$ is a quasi-nonexpansive mapping.

Proposition 2.1 Let $C$ be a closed convex subset of a real Hilbert space H. If $T$ is a quasinonexpansive mapping from $C$ into itself, then $F(T)$ is closed and convex.

Proof Obviously, the continuity of $T$ implies that $F(T)$ is closed. Now, we show that $F(T)$ is convex. For $x, y \in F(T)$ and $t \in(0,1)$, put $z=t x+(1-t) y$. Now, we claim that $z \in F(T)$. In fact,

$$
\begin{aligned}
\|z-T z\|^{2} & =\|z\|^{2}-2\langle z, T z\rangle+\|T z\|^{2} \\
& =\|z\|^{2}-2\langle t x+(1-t) y, T z\rangle+\|T z\|^{2} \\
& =\|z\|^{2}-2 t\langle x, T z\rangle-2(1-t)\langle y, T z\rangle+\|T z\|^{2} \\
& =\|z\|^{2}+t\|x-T z\|^{2}+(1-t)\|y-T z\|^{2}-t\|x\|^{2}-(1-t)\|y\|^{2} \\
& \leq\|z\|^{2}+t\|x-z\|^{2}+(1-t)\|y-z\|^{2}-t\|x\|^{2}-(1-t)\|y\|^{2} \\
& =\|t x+(1-t) y\|^{2}+t\|x-z\|^{2}+(1-t)\|y-z\|^{2}-t\|x\|^{2}-(1-t)\|y\|^{2} \\
& =0,
\end{aligned}
$$

which means that $\|z-T z\|=0$. Hence, $z \in F(T)$ and $F(T)$ is convex.

\section{Main results}

Now, we are in a position to prove our convergence results in this section.

Theorem 3.1 Let $H_{1}, H_{2}, H_{3}$ be real Hilbert spaces. Given two bounded linear operators $A: H_{1} \rightarrow H_{3}, B: H_{2} \rightarrow H_{3}$. Let $\left\{T_{i}\right\}_{i=1}^{N}$ be a finite family of $\rho_{i}$-strictly pseudononspreading mappings of $C$ into itself with $\bigcap_{i=1}^{N} F\left(T_{i}\right) \neq \emptyset$, and Let $\left\{S_{i}\right\}_{i=1}^{N}$ be a finite family of $\tau_{i}$-strictly pseudononspreading mappings of $Q$ into itself with $\bigcap_{i=1}^{N} F\left(S_{i}\right) \neq \emptyset$. Suppose that $U$ is defined by (2.5) which is generated by $T_{1}, T_{2}, \ldots, T_{N}$ and $\lambda_{1}, \lambda_{2}, \ldots, \lambda_{N}$, with $0<\lambda_{i}+\rho_{i}<1$ for every $1,2, \ldots, N$, and suppose that $T$ is defined by (2.5) which is generated by $S_{1}, S_{2}, \ldots, S_{N}$ and $\beta_{1}, \beta_{2}, \ldots, \beta_{N}$, with $0<\beta_{i}+\tau_{i}<1$ for every $1,2, \ldots, N$, respectively. Assume that $U-I$ and 
$T-I$ are demiclosed at the origin. If the solution set $\Gamma$ of (1.4) is nonempty and for small enough $\varepsilon>0$ and $\sigma>0$,

$$
\gamma_{k} \in\left(\varepsilon,(1-\sigma) \min \left\{\frac{\left\|A x_{k}-B y_{k}\right\|^{2}}{\left\|A^{*}\left(A x_{k}-B y_{k}\right)\right\|^{2}}, \frac{\left\|A x_{k}-B y_{k}\right\|^{2}}{\left\|B^{*}\left(A x_{k}-B y_{k}\right)\right\|^{2}}\right\}\right),
$$

then the sequence $\left\{\left(x_{k}, y_{k}\right)\right\}$ generated by Algorithm 1.1 weakly converges to a solution $\left(x^{*}, y^{*}\right)$ of (1.4). Moreover, $\left\|A x_{k}-B y_{k}\right\| \rightarrow 0,\left\|x_{k}-x_{k+1}\right\| \rightarrow 0$, and $\left\|y_{k}-y_{k+1}\right\| \rightarrow 0$ as $k \rightarrow \infty$.

Proof It follows from the condition on $\left\{\gamma_{k}\right\}$ that

$$
\inf _{A x_{k} \neq B y_{k}}\left\{\frac{\left\|A x_{k}-B y_{k}\right\|^{2}}{\left\|A^{*}\left(A x_{k}-B y_{k}\right)\right\|^{2}}-\gamma_{k}\right\}>0
$$

and

$$
\inf _{A x_{k} \neq B y_{k}}\left\{\frac{\left\|A x_{k}-B y_{k}\right\|^{2}}{\left\|B^{*}\left(A x_{k}-B y_{k}\right)\right\|^{2}}-\gamma_{k}\right\}>0
$$

On the other hand, from

$$
\left\|A^{*}\left(A x_{k}-B y_{k}\right)\right\|^{2} \leq\left\|A^{*}\right\|^{2}\left\|A x_{k}-B y_{k}\right\|^{2}
$$

and

$$
\left\|B^{*}\left(A x_{k}-B y_{k}\right)\right\|^{2} \leq\left\|B^{*}\right\|^{2}\left\|A x_{k}-B y_{k}\right\|^{2},
$$

we obtain $\frac{\left\|A x_{k}-B y_{k}\right\|^{2}}{\left\|A^{*}\left(A x_{k}-B y_{k}\right)\right\|^{2}} \geq \frac{1}{\left\|A^{*}\right\|^{2}}$ and $\frac{\left\|A x_{k}-B y_{k}\right\|^{2}}{\left\|B^{*}\left(A x_{k}-B y_{k}\right)\right\|^{2}} \geq \frac{1}{\left\|B^{*}\right\|^{2}}$. Furthermore,

$$
\min \left\{\frac{1}{\left\|A^{*}\right\|^{2}}, \frac{1}{\left\|B^{*}\right\|^{2}}\right\} \leq \min \left\{\frac{\left\|A x_{k}-B y_{k}\right\|^{2}}{\left\|A^{*}\left(A x_{k}-B y_{k}\right)\right\|^{2}}, \frac{\left\|A x_{k}-B y_{k}\right\|^{2}}{\left\|B^{*}\left(A x_{k}-B y_{k}\right)\right\|^{2}}\right\} .
$$

Inequalities (3.1) and (3.2) lead to $\sup _{A x_{k} \neq B y_{k}} \gamma_{k}<+\infty$ and $\left\{\gamma_{k}\right\}$ is bounded.

For $(x, y) \in \Gamma$, by Algorithm 1.1, we obtain

$$
\begin{aligned}
\left\|u_{k}-x\right\|^{2} & =\left\|x_{k}-\gamma_{k} A^{*}\left(A x_{k}-B y_{k}\right)-x\right\|^{2} \\
& =\left\|x_{k}-x\right\|^{2}-2 \gamma_{k}\left(x_{k}-x, A^{*}\left(A x_{k}-B y_{k}\right)\right)+\gamma_{k}^{2}\left\|A^{*}\left(A x_{k}-B y_{k}\right)\right\|^{2} .
\end{aligned}
$$

Notice that

$$
\begin{aligned}
-2\left\langle x_{k}-x, A^{*}\left(A x_{k}-B y_{k}\right)\right\rangle & =-2\left\langle A x_{k}-A x, A x_{k}-B y_{k}\right\rangle \\
& =-\left\|A x_{k}-A x\right\|^{2}-\left\|A x_{k}-B y_{k}\right\|^{2}+\left\|B y_{k}-A x\right\|^{2} .
\end{aligned}
$$

Substituting (3.4) into (3.3), one has

$$
\begin{aligned}
\left\|u_{k}-x\right\|^{2}= & \left\|x_{k}-x\right\|^{2}-\gamma_{k}\left\|A x_{k}-B y_{k}\right\|^{2}-\gamma_{k}\left\|A x_{k}-A x\right\|^{2} \\
& +\gamma_{k}\left\|B y_{k}-A x\right\|^{2}+\gamma_{k}^{2}\left\|A^{*}\left(A x_{k}-B y_{k}\right)\right\|^{2} .
\end{aligned}
$$


Similarly, by Algorithm 1.1, we deduce

$$
\begin{aligned}
\left\|v_{k}-y\right\|^{2}= & \left\|y_{k}-y\right\|^{2}-\gamma_{k}\left\|A x_{k}-B y_{k}\right\|^{2}-\gamma_{k}\left\|B y_{k}-B y\right\|^{2} \\
& +\gamma_{k}\left\|B y-A x_{k}\right\|^{2}+\gamma^{2}\left\|B^{*}\left(A x_{k+1}-B y_{k}\right)\right\|^{2} .
\end{aligned}
$$

Furthermore, adding the two last inequalities, following from the fact $A x=B y$, we have

$$
\begin{aligned}
\left\|u_{k}-x\right\|^{2}+\left\|v_{k}-y\right\|^{2} \leq & \left\|x_{k}-x\right\|^{2}+\left\|y_{k}-y\right\|^{2} \\
& -\gamma_{k}\left(\left\|A x_{k}-B y_{k}\right\|^{2}-\gamma_{k}\left\|A^{*}\left(A x_{k}-B y_{k}\right)\right\|^{2}\right) \\
& -\gamma_{k}\left(\left\|A x_{k}-B y_{k}\right\|^{2}-\gamma_{k}\left\|B^{*}\left(A x_{k}-B y_{k}\right)\right\|^{2}\right) .
\end{aligned}
$$

Next, we will estimate $\left\|x_{k+1}-x\right\|$ and $\left\|y_{k+1}-y\right\|$. It follows from $U$ and $T$ being two quasi-nonexpansive mappings that

$$
\begin{aligned}
\left\|x_{k+1}-x\right\|^{2} & =\left\|a_{k} x_{k}+\left(1-a_{k}\right) U\left(u_{k}\right)-x\right\|^{2} \\
& =\left\|a_{k}\left(x_{k}-x\right)+\left(1-a_{k}\right)\left(U\left(u_{k}\right)-x\right)\right\|^{2} \\
& =a_{k}\left\|x_{k}-x\right\|^{2}+\left(1-a_{k}\right)\left\|U\left(u_{k}\right)-x\right\|^{2}-a_{k}\left(1-a_{k}\right)\left\|U\left(u_{k}\right)-x_{k}\right\|^{2} \\
& \leq a_{k}\left\|x_{k}-x\right\|^{2}+\left(1-a_{k}\right)\left\|u_{k}-x\right\|^{2}-a_{k}\left(1-a_{k}\right)\left\|U\left(u_{k}\right)-x_{k}\right\|^{2}
\end{aligned}
$$

and

$$
\left\|y_{k+1}-y\right\|^{2} \leq a_{k}\left\|y_{k}-y\right\|^{2}+\left(1-a_{k}\right)\left\|v_{k}-y\right\|^{2}-a_{k}\left(1-a_{k}\right)\left\|T\left(v_{k}\right)-y_{k}\right\|^{2} .
$$

Thus, (3.8) and (3.9) lead to

$$
\begin{aligned}
\left\|x_{k+1}-x\right\|^{2}+\left\|y_{k+1}-x\right\|^{2} \leq & a_{k}\left(\left\|x_{k}-x\right\|^{2}+\left\|y_{k}-y\right\|^{2}\right) \\
& +\left(1-a_{k}\right)\left(\left\|u_{k}-x\right\|^{2}+\left\|v_{k}-y\right\|^{2}\right) \\
& -a_{k}\left(1-a_{k}\right)\left(\left\|U\left(u_{k}\right)-x_{k}\right\|^{2}+\left\|T\left(v_{k}\right)-y_{k}\right\|^{2}\right) .
\end{aligned}
$$

Furthermore, it follows from (3.7) that

$$
\begin{aligned}
\left\|x_{k+1}-x\right\|^{2}+\left\|y_{k+1}-x\right\|^{2} \leq & \left\|x_{k}-x\right\|^{2}+\left\|y_{k}-y\right\|^{2} \\
& -\left(1-a_{k}\right) \gamma_{k}\left(\left\|A x_{k}-A x\right\|^{2}-\gamma_{k}\left\|A^{*}\left(A x_{k}-A x\right)\right\|^{2}\right) \\
& -\left(1-a_{k}\right) \gamma_{k}\left(\left\|A x_{k}-B y_{k}\right\|^{2}-\gamma_{k}\left\|B^{*}\left(A x_{k}-B y_{k}\right)\right\|^{2}\right) \\
& -a_{k}\left(1-a_{k}\right)\left(\left\|U\left(u_{k}\right)-x_{k}\right\|^{2}+\left\|T\left(v_{k}\right)-y_{k}\right\|^{2}\right) .
\end{aligned}
$$

Now, setting $\rho_{k}(x, y)=\left\|x_{k}-x\right\|^{2}+\left\|y_{k}-y\right\|^{2}$, one has

$$
\begin{aligned}
\rho_{k+1}(x, y) \leq & \rho_{k}(x, y)-\left(1-a_{k}\right) \gamma_{k}\left(\left\|A x_{k}-A x\right\|^{2}-\gamma_{k}\left\|A^{*}\left(A x_{k}-A x\right)\right\|^{2}\right) \\
& -\left(1-a_{k}\right) \gamma_{k}\left(\left\|A x_{k}-B y_{k}\right\|^{2}-\gamma_{k}\left\|B^{*}\left(A x_{k}-B y_{k}\right)\right\|^{2}\right) \\
& -a_{k}\left(1-a_{k}\right)\left(\left\|U\left(u_{k}\right)-x_{k}\right\|^{2}+\left\|T\left(v_{k}\right)-y_{k}\right\|^{2}\right) .
\end{aligned}
$$


On the other hand, note that

$$
\rho_{k}(x, y)=\left\|x_{k}-x\right\|^{2}+\left\|y_{k}-y\right\|^{2} \geq 0 .
$$

From the assumptions on $\left\{a_{k}\right\}$ and $\left\{\gamma_{k}\right\}$, we see that the sequence $\rho_{k}(x, y)$ being decreasing and lower bounded by 0 , consequently, converges to some finite limit, that is, $\lim _{k \rightarrow \infty} \rho_{k}(x, y)=\rho(x, y)$, which means the sequences $\left\{x_{n}\right\}$ and $\left\{y_{n}\right\}$ are bounded. Thus, we have

$$
\begin{aligned}
& \lim _{k \rightarrow \infty} \gamma_{k}\left(\left\|A x_{k}-B y_{k}\right\|^{2}-\gamma_{k}\left\|A^{*}\left(A x_{k}-B y_{k}\right)\right\|^{2}\right)=0, \\
& \lim _{k \rightarrow \infty} \gamma_{k}\left(\left\|A x_{k}-B y_{k}\right\|^{2}-\gamma_{k}\left\|B^{*}\left(A x_{k}-B y_{k}\right)\right\|^{2}\right)=0
\end{aligned}
$$

and

$$
\lim _{k \rightarrow \infty}\left\|U\left(u_{k}\right)-x_{k}\right\|^{2}=\lim _{k \rightarrow \infty}\left\|T\left(v_{k}\right)-y_{k}\right\|^{2}=0 .
$$

Now, we show that $\lim _{k \rightarrow \infty}\left\|A x_{k}-B y_{k}\right\|=0$. Indeed, as is shown below, we break up the proof by distinguishing two cases.

Case 1. Suppose that there exists $k_{0}$ such that $\frac{\left\|A x_{k}-B y_{k}\right\|^{2}}{\left\|A^{*}\left(A x_{k}-B y_{k}\right)\right\|^{2}} \geq \frac{\left\|A x_{k}-B y_{k}\right\|^{2}}{\left\|B^{*}\left(A x_{k}-B y_{k}\right)\right\|^{2}}$, for all $k \geq k_{0}$, we obtain $\gamma \in\left(\varepsilon,(1-\sigma) \frac{\left\|A x_{k}-B y_{k}\right\|^{2}}{\left\|B^{*}\left(A x_{k}-B y_{k}\right)\right\|^{2}}\right)$. It yields

$$
\begin{aligned}
& \gamma_{k}\left(\left\|A x_{k}-B y_{k}\right\|^{2}-\gamma_{k}\left\|A^{*}\left(A x_{k}-B y_{k}\right)\right\|^{2}\right) \\
& \quad \geq \gamma_{k}\left(\left\|A x_{k}-B y_{k}\right\|^{2}-(1-\sigma) \frac{\left\|A x_{k}-B y_{k}\right\|^{2}}{\left\|B^{*}\left(A x_{k}-B y_{k}\right)\right\|^{2}}\left\|A^{*}\left(A x_{k}-B y_{k}\right)\right\|^{2}\right) \\
& \quad \geq \gamma_{k}\left(\left\|A x_{k}-B y_{k}\right\|^{2}-(1-\sigma) \frac{\left\|A x_{k}-B y_{k}\right\|^{2}}{\left\|A^{*}\left(A x_{k}-B y_{k}\right)\right\|^{2}}\left\|A^{*}\left(A x_{k}-B y_{k}\right)\right\|^{2}\right) \\
& \quad \geq \sigma \varepsilon\left\|A x_{k}-B y_{k}\right\|^{2} .
\end{aligned}
$$

Furthermore, (3.13) leads to

$$
\lim _{k \rightarrow \infty}\left\|A x_{k}-B y_{k}\right\|=0
$$

Since

$$
\left\|A^{*}\left(A x_{k}-B y_{k}\right)\right\|^{2} \leq\left\|A^{*}\right\|^{2}\left\|A x_{k}-B y_{k}\right\|^{2}
$$

and

$$
\left\|B^{*}\left(A x_{k}-B y_{k}\right)\right\|^{2} \leq\left\|B^{*}\right\|^{2}\left\|A x_{k}-B y_{k}\right\|^{2},
$$

we deduce

$$
\lim _{k \rightarrow \infty}\left\|A^{*}\left(A x_{k}-B y_{k}\right)\right\|=\lim _{k \rightarrow \infty}\left\|B^{*}\left(A x_{k}-B y_{k}\right)\right\|=0 .
$$


Conversely, suppose that there exists $k_{1}$ such that $\frac{\left\|A x_{k}-B y_{k}\right\|^{2}}{\left\|A^{*}\left(A x_{k}-B y_{k}\right)\right\|^{2}} \leq \frac{\left\|A x_{k}-B y_{k}\right\|^{2}}{\left\|B^{*}\left(A x_{k}-B y_{k}\right)\right\|^{2}}$, for all $k \geq$ $k_{1}$, following the above process, we obtain the results.

Case 2. Suppose that there does not exist $k_{0}$ such that

$$
\frac{\left\|A x_{k}-B y_{k}\right\|^{2}}{\left\|A^{*}\left(A x_{k}-B y_{k}\right)\right\|^{2}} \geq \frac{\left\|A x_{k}-B y_{k}\right\|^{2}}{\left\|B^{*}\left(A x_{k}-B y_{k}\right)\right\|^{2}}
$$

or

$$
\frac{\left\|A x_{k}-B y_{k}\right\|^{2}}{\left\|A^{*}\left(A x_{k}-B y_{k}\right)\right\|^{2}} \leq \frac{\left\|A x_{k}-B y_{k}\right\|^{2}}{\left\|B^{*}\left(A x_{k}-B y_{k}\right)\right\|^{2}}
$$

for all $k \geq k_{0}$. We can divide the sequence $\frac{\left\|A x_{k}-B y_{k}\right\|^{2}}{\left\|A^{*}\left(A x_{k}-B y_{k}\right)\right\|^{2}}$ into two sequences: one satisfies $\frac{\left\|A x_{k}-B y_{k}\right\|^{2}}{\left\|A^{*}\left(A x_{k}-B y_{k}\right)\right\|^{2}} \geq \frac{\left\|A x_{k}-B y_{k}\right\|^{2}}{\left\|B^{*}\left(A x_{k}-B y_{k}\right)\right\|^{2}}$, which is denoted by $\left\{\frac{\left\|A x_{k_{n}}-B y_{k_{n}}\right\|^{2}}{\left\|A^{*}\left(A x_{k_{n}}-B y_{k_{n}}\right)\right\|^{2}}\right\}$ and the other sequence satisfies $\frac{\left\|A x_{k}-B y_{k}\right\|^{2}}{\left\|A^{*}\left(A x_{k}-B y_{k}\right)\right\|^{2}}<\frac{\left\|A x_{k}-B y_{k}\right\|^{2}}{\left\|B^{*}\left(A x_{k}-B y_{k}\right)\right\|^{2}}$, which is denoted by $\left\{\frac{\left\|A x_{k_{m}}-B y_{k_{m}}\right\|^{2}}{\left\|A^{*}\left(A x_{k_{m}}-B y_{k_{m}}\right)\right\|^{2}}\right\}$. Following the process of Case 1 , we show that the results hold for the subsequences with $k_{n}$ and $k_{m}$. Thus, we obtain $\lim _{k \rightarrow \infty}\left\|A x_{k}-B y_{k}\right\|=0$.

Let us prove that $\left\{x_{k}\right\}$ and $\left\{y_{k}\right\}$ are asymptotically regular. Indeed, since

$$
\left\|u_{k}-x_{k}\right\|=\gamma_{k}\left\|A^{*}\left(A x_{k}-B y_{k}\right)\right\|,
$$

one has

$$
\lim _{k \rightarrow \infty}\left\|u_{k}-x_{k}\right\|=0
$$

Consequently,

$$
\lim _{k \rightarrow \infty}\left\|x_{k+1}-x_{k}\right\|=\lim _{k \rightarrow \infty}\left(1-a_{k}\right)\left\|U\left(u_{k}\right)-x_{k}\right\|=0,
$$

which yields $\left\{x_{k}\right\}$ is asymptotically regular. Similarly, $\lim _{k \rightarrow \infty}\left\|v_{k}-y_{k}\right\|=0$ and $\left\{y_{k}\right\}$ is asymptotically regular, too.

Next, we show that $\left\|u_{k}-U\left(u_{k}\right)\right\| \rightarrow 0$ and $\left\|v_{k}-T\left(v_{k}\right)\right\| \rightarrow 0$ as $k \rightarrow \infty$. Indeed, since

$$
\left\|u_{k}-U\left(u_{k}\right)\right\|=\left\|u_{k}-x_{k}+x_{k}-U\left(u_{k}\right)\right\| \leq\left\|u_{k}-x_{k}\right\|+\left\|x_{k}-U\left(u_{k}\right)\right\|,
$$

(3.15) and (3.17) mean that $\lim _{k \rightarrow \infty}\left\|u_{k}-U\left(u_{k}\right)\right\|=0$. In the same way as above, we can also show that $\left\|v_{k}-T\left(v_{k}\right)\right\| \rightarrow 0$ as $k \rightarrow \infty$.

Taking $\left(x^{*}, y^{*}\right) \in \omega_{w}\left(x_{k}, y_{k}\right)$, from $\lim _{k \rightarrow \infty}\left\|u_{k}-x_{k}\right\|=0$ and $\lim _{k \rightarrow \infty}\left\|v_{k}-y_{k}\right\|=0$, we obtain $x \in \omega_{w}\left(x_{k}\right)$ and $y \in \omega_{w}\left(y_{k}\right)$. Combining with the demiclosednesses of $U-I$ and $T-I$ at 0 , one has

$$
\lim _{k \rightarrow \infty}\left\|U\left(u_{k}\right)-u_{k}\right\|=\lim _{k \rightarrow \infty}\left\|T\left(v_{k}\right)-v_{k}\right\|=0,
$$

which yields $U x^{*}=x^{*}$ and $T y^{*}=y^{*}$. Thus, $x^{*} \in F(U)$ and $y^{*} \in F(T)$. On the other hand, $A x^{*}-B y^{*} \in \omega_{w}\left(A x_{k}-B y_{k}\right)$ and lower semicontinuity of the norm imply that

$$
\left\|A x^{*}-B y^{*}\right\| \leq \lim _{k \rightarrow \infty} \inf \left\|A x_{k}-B y_{k}\right\|=0,
$$

hence $\left(x^{*}, y^{*}\right) \in \Gamma$. 
Finally, we will show the uniqueness of the weak cluster points of $\left\{x_{k}\right\}$ and $\left\{y_{k}\right\}$. Indeed, let $\bar{x}, \bar{y}$ be other weak cluster points of $\left\{x_{k}\right\}$ and $\left\{y_{k}\right\}$, respectively. From the definition of $\rho_{k}(x, y)$, we have

$$
\begin{aligned}
\rho_{k}\left(x^{*}, y^{*}\right)= & \left\|x_{k}-x^{*}\right\|^{2}+\left\|y_{k}-y^{*}\right\|^{2} \\
= & \left\|x_{k}-\bar{x}\right\|^{2}+\left\|\bar{x}-x^{*}\right\|^{2}+2\left\langle x_{k}-\bar{x}, \bar{x}-x^{*}\right\rangle \\
& +\left\|y_{k}-\bar{y}\right\|^{2}+\left\|\bar{y}-y^{*}\right\|^{2}+2\left\langle y_{k}-\bar{y}, \bar{y}-y^{*}\right\rangle \\
= & \rho_{k}(\bar{x}, \bar{y})+\left\|\bar{x}-x^{*}\right\|^{2}+\left\|\bar{y}-y^{*}\right\|^{2} \\
& +2\left\langle x_{k}-\bar{x}, \bar{x}-x^{*}\right\rangle+2\left\langle y_{k}-\bar{y}, \bar{y}-y^{*}\right\rangle .
\end{aligned}
$$

Without loss of generality, we may assume that $x_{k} \rightarrow \bar{x}, y_{k} \rightarrow \bar{x}$, and then

$$
\rho\left(x^{*}, y^{*}\right)=\rho(\bar{x}, \bar{y})+\left\|\bar{x}-x^{*}\right\|^{2}+\left\|\bar{y}-y^{*}\right\|^{2} .
$$

Reversing the role of $\left(x^{*}, y^{*}\right)$ and $(\bar{x}, \bar{y})$, we obtain

$$
\rho(\bar{x}, \bar{y})=\rho\left(x^{*}, y^{*}\right)+\left\|x^{*}-\bar{x}\right\|^{2}+\left\|y^{*}-\bar{y}\right\|^{2} .
$$

Equations (3.19) and (3.20) yield

$$
\left\|x^{*}-\bar{x}\right\|^{2}+\left\|y^{*}-\bar{y}\right\|^{2}=0
$$

which means $x^{*}=\bar{x}$ and $y^{*}=\bar{y}$. Hence, the sequence $\left\{\left(x_{k}, y_{k}\right)\right\}$ weakly converges to a solution of the problem (1.4), which completes the proof.

The following conclusions can be obtained from Theorem 3.1 immediately.

Theorem 3.2 Let $H_{1}, H_{2}, H_{3}$ be real Hilbert spaces. Given two bounded linear operators $A: H_{1} \rightarrow H_{3}, B: H_{2} \rightarrow H_{3}$. Let $U$ be a $\rho$-strictly pseudononspreading mapping of $C$ into itself and Let $T$ be a $\tau$-strictly pseudononspreading mapping of $Q$ into itself. Assume that $U-I$ and $T-I$ are demiclosed at the origin. If the solution set $\Gamma$ of (1.4) is nonempty and for small enough $\varepsilon>0$ and $\sigma>0$,

$$
\gamma_{k} \in\left(\varepsilon,(1-\sigma) \min \left\{\frac{\left\|A x_{k}-B y_{k}\right\|^{2}}{\left\|A^{*}\left(A x_{k}-B y_{k}\right)\right\|^{2}}, \frac{\left\|A x_{k}-B y_{k}\right\|^{2}}{\left\|B^{*}\left(A x_{k}-B y_{k}\right)\right\|^{2}}\right\}\right)
$$

then the sequence $\left\{\left(x_{k}, y_{k}\right)\right\}$ generated by Algorithm 1.1 weakly converges to a solution $\left(x^{*}, y^{*}\right)$ of (1.4). Moreover, $\left\|A x_{k}-B y_{k}\right\| \rightarrow 0,\left\|x_{k}-x_{k+1}\right\| \rightarrow 0$, and $\left\|y_{k}-y_{k+1}\right\| \rightarrow 0$ as $k \rightarrow \infty$.

Theorem 3.3 Let $H_{1}, H_{2}, H_{3}$ be real Hilbert spaces. Given two bounded linear operators $A: H_{1} \rightarrow H_{3}, B: H_{2} \rightarrow H_{3}$. Let $U$ be a nonspreading mapping of $C$ into itself and let $T$ be a nonspreading mapping of $Q$ into itself. Assume that $U-I$ and $T-I$ are demiclosed at the origin. If the solution set $\Gamma$ of (1.4) is nonempty and for small enough $\varepsilon>0$ and $\sigma>0$,

$$
\gamma_{k} \in\left(\varepsilon,(1-\sigma) \min \left\{\frac{\left\|A x_{k}-B y_{k}\right\|^{2}}{\left\|A^{*}\left(A x_{k}-B y_{k}\right)\right\|^{2}}, \frac{\left\|A x_{k}-B y_{k}\right\|^{2}}{\left\|B^{*}\left(A x_{k}-B y_{k}\right)\right\|^{2}}\right\}\right),
$$


then the sequence $\left\{\left(x_{k}, y_{k}\right)\right\}$ generated by Algorithm 1.1 weakly converges to a solution $\left(x^{*}, y^{*}\right)$ of (1.4). Moreover, $\left\|A x_{k}-B y_{k}\right\| \rightarrow 0,\left\|x_{k}-x_{k+1}\right\| \rightarrow 0$, and $\left\|y_{k}-y_{k+1}\right\| \rightarrow 0$ as $k \rightarrow \infty$.

\section{Applications}

We now pay attention to applying our simultaneous iterative algorithms to some convex and nonlinear analysis notions; see, for example, [21].

\subsection{Split feasibility problem}

Let $C$ and $Q$ be nonempty closed convex subset of real Hilbert spaces $H_{1}$ and $H_{2}$, respectively. The split feasibility problem (SFP) is to find a point

$x \in C$ such that $A x \in Q$,

where $A: H_{1} \rightarrow H_{2}$ is a bounded linear operator. The SFP was first introduced by Censor and Elfving [22] for modeling inverse problems which arise from phase retrievals and in medical image reconstruction [23].

If $B=I, H_{2}=H_{3}$, then Algorithm 1.1 becomes:

\section{Algorithm 4.1}

$$
\left\{\begin{array}{l}
u_{k}=x_{k}-\gamma_{k} A^{*}\left(A x_{k}-y_{k}\right), \\
x_{k+1}=a_{k} x_{k}+\left(1-a_{k}\right) U\left(u_{k}\right), \\
v_{k}=y_{k}+\gamma_{k}\left(A x_{k}-y_{k}\right), \\
y_{k+1}=a_{k} y_{k}+\left(1-a_{k}\right) T\left(v_{k}\right),
\end{array}\right.
$$

where the stepsize $\gamma_{k}$ is chosen by (1.6). If $A x_{k}=y_{k}$, then $\left(x_{k}, y_{k}\right)=\left(x_{k+1}, y_{k+1}\right)$ is a solution of the problem (4.1) and the iterative process stops. Otherwise, we set $k:=k+1$ and go on to (4.1) to evaluate the next iterate $\left(x_{k+2}, y_{k+2}\right)$.

Furthermore, if $U=P_{C}$ and $T=P_{Q}$, then we obtain the following simultaneous iterative algorithm for solving SFP (4.1).

\section{Algorithm 4.2}

$$
\left\{\begin{array}{l}
u_{k}=x_{k}-\gamma_{k} A^{*}\left(A x_{k}-y_{k}\right), \\
x_{k+1}=a_{k} x_{k}+\left(1-a_{k}\right) P_{C}\left(u_{k}\right), \\
v_{k}=y_{k}+\gamma_{k}\left(A x_{k}-y_{k}\right), \\
y_{k+1}=a_{k} y_{k}+\left(1-a_{k}\right) P_{Q}\left(v_{k}\right),
\end{array}\right.
$$

where the stepsize $\gamma_{k}$ is chosen by (1.6). If $A x_{k}=y_{k}$, then $\left(x_{k}, y_{k}\right)=\left(x_{k+1}, y_{k+1}\right)$ is a solution of the problem (4.1) and the iterative process stops. Otherwise, we set $k:=k+1$ and go on to (4.3) to evaluate the next iterate $\left(x_{k+2}, y_{k+2}\right)$.

\subsection{Variational problems via resolvent mappings}

Given a maximal monotone operator $M: H_{1} \rightarrow 2^{H_{1}}$, it is well known that its associated resolvent mapping, $J_{\mu}^{M}=(I+\mu M)^{-1}$, is quasi-nonexpansive and $0 \in M(x) \Leftrightarrow x=J_{\mu}^{M}(x)$, which implies that zeroes of $M$ are exactly fixed points of its resolvent mapping. If $U^{k}=J_{\mu}^{M}$ 
and $T^{k}=J_{v}^{N}$, where $N: H_{2} \rightarrow 2^{H_{2}}$ is another maximal monotone operator, the problem under consideration is nothing but

$$
\text { find } x^{*} \in M^{-1}(0), y^{*} \in N^{-1}(0) \text { such that } A x^{*}=B y^{*} \text {, }
$$

and the algorithm is applied to the following form.

\section{Algorithm 4.3}

$$
\left\{\begin{array}{l}
u_{k}=x_{k}-\gamma_{k} A^{*}\left(A x_{k}-B y_{k}\right), \\
x_{k+1}=a_{k} x_{k}+\left(1-a_{k}\right) J_{\mu}^{M} u_{k}, \\
v_{k}=y_{k}+\beta_{k} B^{*}\left(A x_{k}-B y_{k}\right), \\
y_{k+1}=a_{k} y_{k}+\left(1-a_{k}\right) J_{v}^{N} v_{k},
\end{array}\right.
$$

where the stepsize $\gamma_{k}$ is chosen by (1.6). If $A x_{k}-B y_{k}=0$, then $\left(x_{k}, y_{k}\right)=\left(x_{k+1}, y_{k+1}\right)$ is a solution of the problem (4.4) and the iterative process stops. Otherwise, we set $k:=k+1$ and go on to (4.5) to evaluate the next iterate $\left(x_{k+2}, y_{k+2}\right)$.

\section{Competing interests}

The authors declare that there is no conflict of interests regarding the publication of this paper

\section{Authors' contributions}

The authors have contributed in this work on an equal basis. All authors read and approved the final manuscript.

\section{Acknowledgements}

We thank Prof. Yiju Wang for his careful reading of the manuscript and thank the anonymous referees and the editor for their constructive comments and suggestions, which greatly improved this article. This project is supported by the Natural Science Foundation of China (Grant Nos. 11401438, 11171180, 11171193, 11126233), and Project of Shandong

Province Higher Educational Science and Technology Program (Grant No. J14LI52)

\section{Received: 1 July 2014 Accepted: 8 October 2014 Published: 05 Jan 2015}

\section{References}

1. Aleyner, A, Reich, S: Block-iterative algorithms for solving convex feasibility problems in Hilbert and in Banach spaces. J. Math. Anal. Appl. 343(1), 427-435 (2008)

2. Bauschke, HH, Borwein, JM: On projection algorithms for solving convex feasibility problems. SIAM Rev. 38(3) 367-426 (1996)

3. Byrne, C: A Unified Treatment of Some Iterative Algorithms in Signal Processing and Image Reconstruction. Dekker, New York (1984)

4. Masad, E, Reich, S: A note on the multiple-set split convex feasibility problem in Hilbert space. J. Nonlinear Convex Anal. 8, 367-371 (2007)

5. Yao, Y, Chen, R, Marino, G, Liou, YC: Applications of fixed point and optimization methods to the multiple-sets split feasibility problem. J. Appl. Math. 2012, Article ID 927530 (2012)

6. Qu, B, Xiu, N: A note on the CQ algorithm for the split feasibility problem. Inverse Probl. 21, 1655-1665 (2005)

7. Qu, B, Xiu, N: A new halfspace-relaxation projection method for the split feasibility problem. Linear Algebra Appl. 428(5), 1218-1229 (2008)

8. Xu, HK: Iterative methods for the split feasibility problem in infinite-dimensional Hilbert spaces. Inverse Probl. 26(10), 5018-5034 (2010)

9. Censor, Y, Elfving, T, Kopf, N, Bortfled, T: The multi-sets split feasibility problem and it applications to inverse problems Inverse Probl. 21, 2071-2084 (2005)

10. Yang, Q: The relaxed CQ algorithm for solving the split feasibility problem. Inverse Probl. 20, 1261-1266 (2004)

11. Zhao, J, Yang, Q: Several solution methods for the split feasibility problem. Inverse Probl. 21, 1791-1799 (2005)

12. Moudafi, A: Alternating CQ-algorithm for convex feasibility and split fixed-point problems. J. Nonlinear Convex Anal. $15,809-818(2014)$

13. Attouch, $\mathrm{H}$, Bolte, J, Redont, $\mathrm{P}$, Soubeyran, A: Alternating proximal algorithms for weakly coupled minimization problems. Applications to dynamical games and PDEs. J. Convex Anal. 15, 485-506 (2008)

14. Censor, Y, Bortfeld, T, Martin, B, Trofimov, A: A unified approach for inversion problems in intensity-modulated radiation therapy. Phys. Med. Biol. 51, 2353-2365 (2006)

15. Moudafi, A, Al-Shemas, E: Simultaneous iterative methods for split equality problems and applications. Trans. Math Program. Appl. 1, 1-11 (2013) 
16. Lopez, G, Martin-Marquez, V, Wang, F, Xu, H: Solving the split feasibility problem without prior knowledge of matrix norms. Inverse Probl. 27, 085004 (2012)

17. Zhao, J, Zhang, J, Yang, Q: A simple projection method for solving the multiple-sets split feasibility problem. Inverse Probl. Sci. Eng. 21, 537-546 (2013)

18. Maruster, S, Popirlan, C: On the Mann-type iteration and convex feasibility problem. J. Comput. Appl. Math. 24, 390-396 (2008)

19. Chang, SS: Some problems and results in the study of nonlinear analysis. Nonlinear Anal. 30(7), 4197-4208 (1997)

20. Kangtunyakarn, A, Suantai, S: A new mapping for finding common solutions of equilibrium problems and fixed point problems of finite family of nonexpansive mappings. Nonlinear Anal. 71, 4448-4460 (2009)

21. Rockafellar, RT, Wets, R: Variational Analysis. Grundlehren der Mathematischen Wissenschafte, vol. 317. Springer, Berlin (1998)

22. Censor, Y, Elfving, T: A multiprojection algorithm using Bregman projections in a product space. Numer. Algorithms 8 221-239 (1994)

23. Byrne, C: Iterative oblique projection onto convex subsets and the split feasibility problem. Inverse Probl. 18, 441-453 (2002)

10.1186/1687-1812-2015-1

Cite this article as: Che and Li: A simultaneous iterative method for split equality problems of two finite families of strictly pseudononspreading mappings without prior knowledge of operator norms. Fixed Point Theory and Applications 2015, 2015:1

\section{Submit your manuscript to a SpringerOpen ${ }^{\circ}$ journal and benefit from:}

- Convenient online submission

- Rigorous peer review

- Immediate publication on acceptance

- Open access: articles freely available online

- High visibility within the field

- Retaining the copyright to your article 